\title{
Towards self-doping multimetal porphyrin systems
}

\author{
Richard Goddard, ${ }^{\mathrm{a}}$ Ralf Tonner ${ }^{\mathrm{b}}$ and Manfred T. Reetz ${ }^{\mathrm{a}, \mathrm{b}}$
}

a. Max-Planck-Institut für Kohlenforschung, Kaiser-Wilhelm-Platz 1, 45470 Mülheim/Germany.

b. $\quad$ Fachbereich Chemie, Philipps-Universität Marburg, Hans-Meerwein-Str. 4, 35032 Marburg/Germany.

† Footnotes relating to the title and/or authors should appear here.

Electronic Supplementary Information (ESI) available: Experimental details, and additional spectroscopic and computational results. CCDC 1970038. For ESI and crystallographic data in CIF see DOI: $10.1039 / x 0 x x 00000 x$ 
Heating $\mathrm{Cu}(\mathrm{II})$ tetraphenylporphyrin (TPPCu) with chromocene at $12{ }^{\circ} \mathrm{C}$ affords the crystalline self-doped multimetal porphyrin system TPPCu/TPPCr in good yield. The Xray structural analysis reveals a random configuration of TPPCu and TPPCr with a $\mathrm{Cu}: \mathrm{Cr}$ ratio of about 71:29 \%. Exploratory DFT calculations indicate significant electron transfer in a hypothetical cationic TPPCu/TPPRu system, in contrast to TPPCu/TPPCr.

Chemists have broadened the chemical scope of naturally occurring metalloporphyrins significantly by synthesizing derivatives with sterically and electronically different substituents and different transition metals, ${ }^{1}$ and/or by focusing in the same way on structurally related metallophthalocyanines ${ }^{1,2}$ or metallocorroles. ${ }^{3}$ This ongoing research is driven, inter alia, by the realization that many of these materials exhibit unexpected electronic characteristics such as unusual magnetic or electrically conducting properties, ${ }^{1-3}$ including surface chemistry of metalloporphyrins and metallophthalocanines. ${ }^{4}$ Catalysis using metalloporphyrins and structurally related complexes also continues to be an exciting research area. ${ }^{1-3,5}$ The key observation by Marks that appropriate oxidants functioning as dopants greatly influence the magnitude of such properties in metallophthalocyanines established a convenient way to influence metallo-systems in general, ${ }^{6}$ a strategy that had been reported earlier by Shirakawa, MacDiarmid and Heeger for increasing the electrical conductivity of polyacetylene. ${ }^{7}$

Inspired by these developments, we envisioned a system consisting of a metalloporphyrin harboring metal $X$ and a second metalloporphyrin with metal $Y$, the latter serving as a reducing or oxidizing dopant. Here we report initial steps toward implementing such a self-doping metalloporphyrin system. Rather than mixing two different metalloporphyrins to achieve this end, commercially available $\mathrm{Cu}(\mathrm{II})$ tetraphenyl-porphyrin (TPPCu) in benzonitrile was heated in a benzonitrile solution of chromocene at $120{ }^{\circ} \mathrm{C}$ for 6 hours (Scheme 1). ${ }^{8}$ Following filtration and storage of the solution for several days at room temperature, dark violet quadratic-bipyramidal crystals formed, which were collected, washed with hexane and dried under high vacuum. 


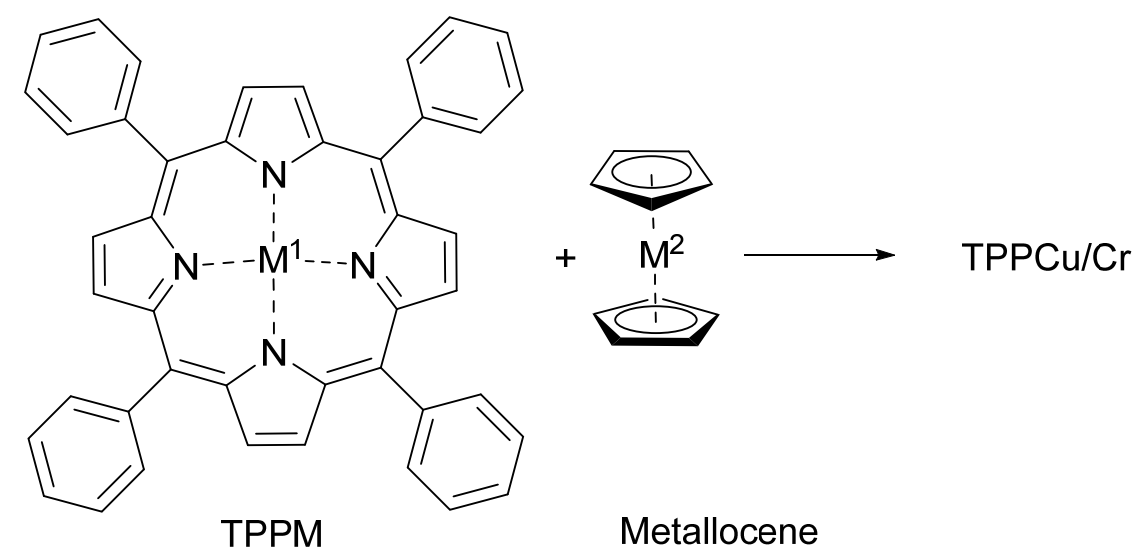

Scheme 1. Synthesis of the mixed metallo-tetraphenyl-porphyrin system TPPCu/Cr by heating TPPM $\left(\mathrm{M}^{1}=\mathrm{Cu}\right)$ with chromocene (metallocene with $\mathrm{M}^{2}=\mathrm{Cr}$ ) at $120^{\circ} \mathrm{C}$.

Elemental analysis of a crudely purified sample revealed an approximate composition of $\mathrm{Cu}: \mathrm{Cr}=58: 42$ (C: 78.20\%; H: 4.55\%; N: 8.55\%; Cu: 5.32\%; Cr: 3.19\%). In the IR-spectrum, the known characteristic bands of chromocene at 1090, 990 and $770 \mathrm{~cm}^{-1}$ were absent (see Supp. Info, Fig. S3), showing that chromocene had not survived the reaction conditions, and that $\mathrm{Cr}$ had possibly embedded itself in the porphyrin ligand. We therefore surmised that the unknown crystalline material is indeed the mixed metalloporphyrin system TPPCu/Cr, formed by a new type of metal/ligand substitution reaction via a hitherto unknown mechanism.

The crystal structure analysis of a purified sample reveals a random configuration of TPPCu and TPPCr with a $\mathrm{Cu}: \mathrm{Cr}$ ratio of about 71:29 (Fig. 1). The mixed metal porphyrin crystallizes in the tetragonal space group $142 \mathrm{~d}$ and is isostructural with one crystal form of the parent metal-free 5,10,15,20-tetraphenyl porphyrin TPP ${ }^{9}$ and 5,10,15,20tetraphenylporphyrin complexes of divalent iron, ${ }^{10}$ cobalt, $^{11}$ nickel, ${ }^{12}$ copper, ${ }^{12 a, 13}$ palladium ${ }^{12 a}$ and platinum. ${ }^{14}$ In all these latter structures, the metal is strictly square planar coordinated, and the porphyrin ligand adopts a ruf (ruffled) conformation. In TPPCu/Cr the $\mathrm{Cu} / \mathrm{Cr}-\mathrm{N}$ distances are 1.987(2) $\AA$ and the shortest distance between the metal atoms is $8.295(1) \AA$. For the complexes that crystallize in this space group the shortest metal to metal distances are 8.250/8.317 $\AA \mathrm{Fe}(\mathrm{II}),{ }^{10} 8.300 / 8.236 / 8.310 \AA$ $\mathrm{Co}(\mathrm{II}),{ }^{11} 8.290 / 8.307 \AA \mathrm{Ni}(\mathrm{II}),{ }^{12} 8.289 / 8.237 / 8.286 / 8.245 \AA \mathrm{Cu}(\mathrm{II}),{ }^{12 a, 13} 8.315 \AA \mathrm{Pd}(\mathrm{II}),{ }^{12 a}$ $8.308 \AA \mathrm{Pt}(\mathrm{II}) .{ }^{14}$ Clearly, the nature of the metal does not appear to have a significant effect on the crystal packing. 

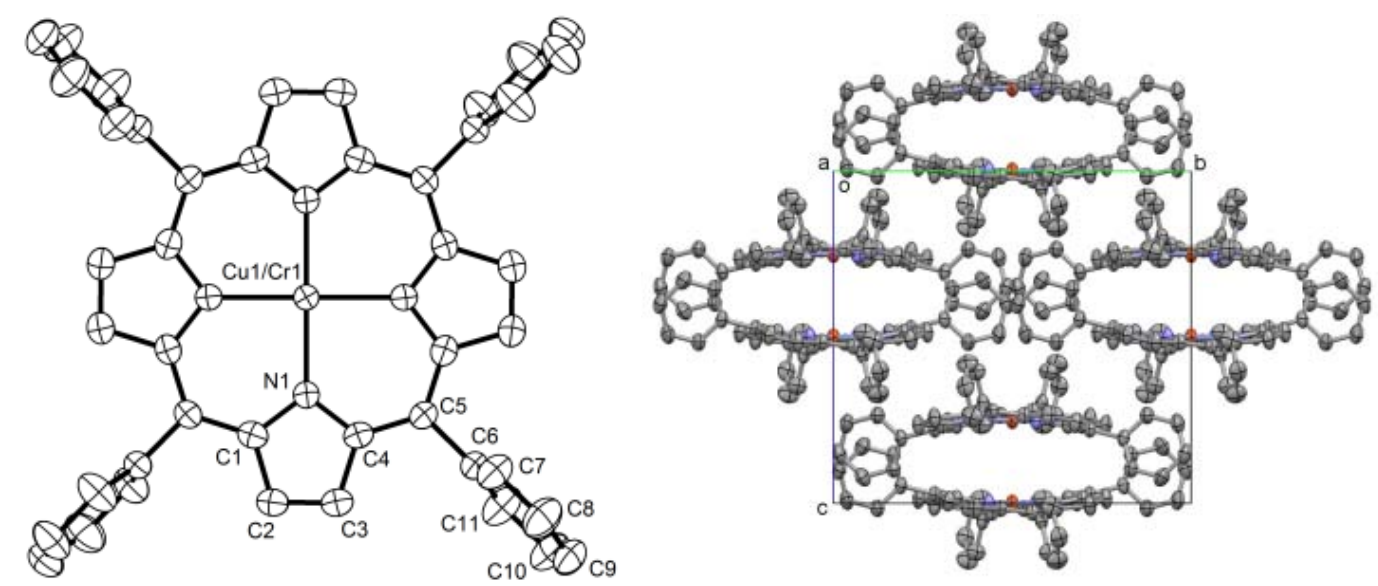

Figure 1. Structure of TPPCu/Cr in the solid state, showing the atom numbering scheme (left) and a view along the $a$ axis of the tetragonal unit cell (right). $\mathrm{H}$ atoms have been removed for clarity.

This packing arrangement is, however, not unique for 5,10,15,20-tetraphenyl porphyrin metal complexes. TPP metal complexes also crystallize without solvents in triclinic $P 1$ [Li radical, ${ }^{15} \mathrm{Zn}(\mathrm{II}),{ }^{16} \mathrm{Pt}(\mathrm{II}),{ }^{17} \mathrm{Cd}(\mathrm{II}),{ }^{18} \mathrm{Ag}(\mathrm{II})^{16}$ and remarkably $\mathrm{Au}(\mathrm{II}),{ }^{19}$ in which the metal adopts the less common +2 oxidation state and where the shortest intermolecular gold to gold distance is $6.17 \AA]$, monoclinic $P 2_{1} / \mathrm{n}\left[\mathrm{Cu}(\mathrm{II}),{ }^{20} \mathrm{Zn}(\mathrm{II})^{21}\right]$ and orthorhombic $P 2_{1} 2_{1} 2_{1}$ [Zn(II) $\left.{ }^{12 a}\right]$ space groups, whereby it is notable that TPPAg also forms a solid solution with the parent metal-free porphyrin which crystallizes in the same space group $P 1 .^{22}$ The two compounds appear to be perfectly miscible. ${ }^{23}$

In contrast, the crystal structure of TPPCr has only been reported as a toluene solvate. ${ }^{24}$ In this case, the macrocycle is almost planar, with the phenyl groups bent circa $70^{\circ}$ out of the plane. The space group $\left(P 2_{1} / \mathrm{c}\right.$, no. 14$)$ requires only that the molecule exhibits a centre of symmetry. ${ }^{24}$ In addition, TPPCu, ${ }^{25}$ TPPGe $^{26}$ and TPPAg $^{27}$ are reported to crystallize in the space group $14 / \mathrm{m}$, though the presence of voids in the crystal structures indicate that the structures may not be solvent free.

Whether a metal atom lies in the plane of the porphyrin ring affects the crystal packing and largely depends on the size of the metal. The average covalent radii of metals derived from the Cambridge Structural Database give an indication of the size of the metal. Radii are for lithium 1.28(7) $\AA$, chromium 1.39(5) $\AA$, iron $1.32(3) / 1.52(6) \AA$ (low/high spin), cobalt 1.26(3)/1.50(7) $\AA$ (low/high spin), nickel 1.24(4) $\AA$, copper 1.32(4) $\AA$, palladium $1.39(6) \AA$, platinum $1.36(5) \AA$, and cadmium $1.44(9) \AA .{ }^{28}$ Interestingly, in TPPCd the cadmium atom appears too large to fit in the plane of the macrocycle and the porphyrin adopts a domed-shaped conformation in the pure solid state with the $\mathrm{Cd}$ 
atoms lying 0.578(6) $\AA$ out of the mean plane through the $\mathrm{N}$ atoms of the porphyrin ring. ${ }^{18} \mathrm{~A}$ similar structure is observed for TPPGe, but the germanium atom is disordered over two positions, one below and one above the plane of the macrocycle. ${ }^{26}$

In principle, short metal-metal distances are possible for metal porphyrin structures, causing an additional disturbance to the crystal packing. For the TPPCd the shortest Cd...Cd distance is $4.14 \AA .{ }^{18}$ For a $\beta, \beta^{\prime}$-linked porphyrin-chlorin di-copper heterodimer, the shortest intermolecular $\mathrm{Cu} . . . \mathrm{Cu}$ distance is $4.88 \AA \AA^{29}$ For solid TPPZn the intermolecular Zn...Zn distance is $6.44 \AA \AA^{16}$ The shortest metal-metal distance for neutral metal porphyrins in general has been observed for [TPPMo] $]_{2}$ benzene decalin solvate, where the Mo...Mo distance is $2.24 \AA .^{30}$

The mixed metal tetraphenyl porphyrin TPPCu/Cr described here was synthesized by reacting copper tetraphenyl porphyrin with chromocene as a source for $\mathrm{Cr}$. This contrasts with a report of a crystal of cobalt tetraphenylporphyrin and dibenzene chromium, in which the two molecules coexist. ${ }^{31}$

The key feature of the TPPCu/Cr crystal structure is that the two metal centers are spatially fairly far away from each other (ca. 8.3 Å), which means that electron transfer and thus a possible dopant effect may be impeded. Indeed, magnetic measurements did not reveal definitive electron transfer. ${ }^{8}$ The present system is thus quite different from polymeric metallophthalocyanine in which the externally doped phthalocyanine entities are nicely positioned for smooth electron transfer within the stack. ${ }^{6}$

In order to provide an initial theoretical understanding of our material, exploratory density functional theory (DFT) calculations at the GGA level of approximating exchangecorrelation effects including a semiempirical dispersion correction scheme (BP86D3/def2-SVP) were performed. ${ }^{32}$ Computational investigations on an unrelaxed dimer model of the co-crystal TPPCu/Cr (Figure 2a, b) support the notion of negligible dopant effect as indicated by a simple atomic partial charge scheme (natural population analysis, NPA). ${ }^{32 f}$ The two neighboring molecules, extracted from the unit cell and chosen to contain $\mathrm{Cu}$ and $\mathrm{Cr}$, respectively, indeed show slightly less positive partial charges at the metal centers compared to the optimized monomer structures TPPCu and TPPCr (Table 1). However, this is due to the increase in $\mathrm{M}-\mathrm{N}$ bond distance in the optimized complexes (2.034 and $2.040 \AA$ for TPPCu and TPPCr) compared to the unrelaxed dimer (1.986 $\AA$ ), since both metal-ligand complexes remain overall neutral in line with the long M1-M2 distance of $8.3 \AA$. One key question regarding these complexes is their electronic ground state. From previous work, we expect TPPCU/TPPCr 
to contain $\mathrm{Cu}(\mathrm{II})\left(\mathrm{d}^{9}\right)$ and $\mathrm{Cr}(\mathrm{II})\left(\mathrm{d}^{4}\right)$. While TPPCu shows a low-spin doublet ground state $^{1 b, 1 c}$ porphyrin complexes of $\mathrm{Cr}$ are discussed as paramagnetic, ${ }^{1 \mathrm{~d}}$ indicating a highspin configuration (quintet), but can possibly become low-spin in the solid state. ${ }^{1 \mathrm{e}}$ This is reflected in our analysis showing the high-spin configuration to be energetically preferred for TPPCr (intermediate spin: $+25.3 \mathrm{kcal} \mathrm{mol}^{-1}$, low spin: $+22.9 \mathrm{kcal} \mathrm{mol}^{-1}$ ), while for the unrelaxed dimer structure high and intermediate spin are isoenergetic (low spin: $+22.9 \mathrm{kcal} \mathrm{mol}^{-1}$ ). This demonstrates the limits of our exploratory computational method and indicates the need for higher-level calculations with multireference wavefunction-based approaches to settle this question. Nevertheless, we can suggest a possible strategy to achieve the desired goal. Although, the TPPCu/TPPCr complex shows indications for an insignificant charge transfer, a related model system seems more promising.

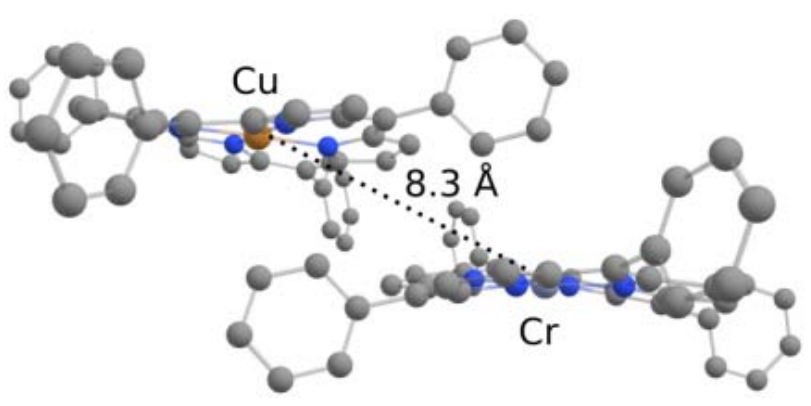

a)

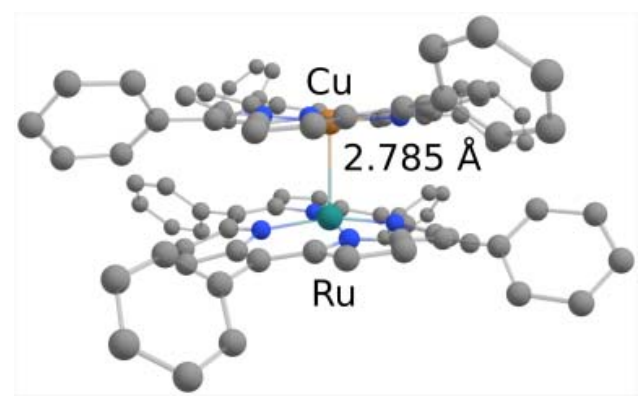

c)

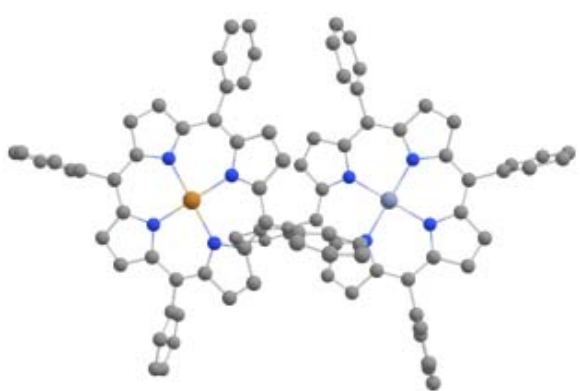

b)

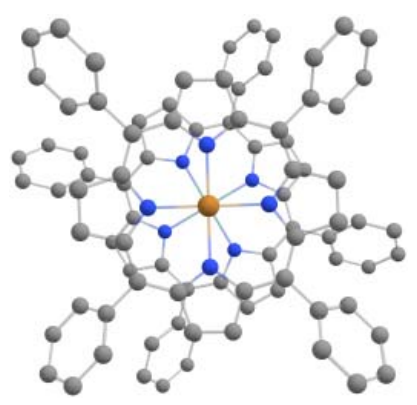

d)

Figure 2. Extracted dimer of experimental structure of TPPCu/TPPCr in a) side and b) top view. Computationally optimized (BP86-D3/def2-SVP) dimer for cationic TPPCu/TPPRu model system in c) side and d) top view. 
If we substitute $\mathrm{Cr}(\mathrm{II})$ by more redox-active $\mathrm{Ru}(\mathrm{II})$, our computations show that cationic complexes result that have not been isolated yet, but show the features we propose (Figure 2). With the parent porphyrin (Por) as ligand, a high-spin model complex PorCu/PorRu results with a stacked arrangement of the metal atoms in close contact $(2.765 \AA)$ and moderate charge transfer of 0.41 e (PorRu $\rightarrow$ PorCu) indicated by NPA. Even the experimentally more relevant TPPCU/TPPRu complex shows a close M1-M2 distance of $2.785 \AA$ and a charge transfer of $0.44 \mathrm{e}$. This is possible due to the staggered arrangement of the phenyl groups (Figure $2 \mathrm{~d}$ ) that enables close packing and makes this structure a promising synthetic target.

With regard to the suitability of TPPRu to form a complex with other metal tetraphenylporphyrins, it is worth noting that of the 266 crystal structures of ruthenium porphyrin complexes in the Cambridge Structural Database (CSD, December 2019), ${ }^{33}$ all contain additional ligands attached to the metal, reflecting the relatively high Lewis acidity of ruthenium when situated in a porphyrin ring. Complete transfer of one electron has been observed for alkali metal metalloporphyrin complexes of formally $\mathrm{Co}(\mathrm{I}),{ }^{34} \mathrm{Fe}(\mathrm{I})$ and $\mathrm{Fe}(0) \cdot{ }^{35}$

The computations thus point toward possible design principles for self-doping by choosing redox-active metal centers and "stackable" ligands.

Table 1. Key structural parameters and partial charges for monomeric and dimeric metalloporphyrin complexes from computations (BP86-D3/def2-SVP). ${ }^{\text {[a] }}$

\begin{tabular}{|c|c|c|c|c|c|}
\hline $\begin{array}{l}\mathrm{LM}^{1} \\
\mathrm{LM}^{2}\end{array}$ & $\begin{array}{c}\text { TPPCu } \\
\text { TPPCr }^{[b]}\end{array}$ & $\begin{array}{c}\text { TPPCu } \\
-\end{array}$ & $\begin{array}{c}\text { TPPCr } \\
-\end{array}$ & $\begin{array}{l}\text { PorCu } \\
\text { PorRu }\end{array}$ & $\begin{array}{l}\text { TPPCu } \\
\text { TPPRu }\end{array}$ \\
\hline$d\left(M^{1}-N\right)$ & 1.986 & 2.034 & 2.040 & 2.037 & $2.037-2.051$ \\
\hline$d\left(M^{2}-N\right)$ & 1.986 & & & 2.041 & $2.042-2.043$ \\
\hline$<(\mathrm{N}-\mathrm{N}-\mathrm{N}-\mathrm{N})$ & 3.1 & 0.4 & 0.3 & 0.0 & $0.6 / 2.6$ \\
\hline$d(M-M)$ & 8.3 & - & - & 2.765 & 2.785 \\
\hline$q\left(M^{1} / M^{2}\right)^{[c]}$ & $+0.85 /+0.77$ & +0.89 & +0.85 & $+0.86 /+0.64$ & $+0.86 /+0.60$ \\
\hline q(L@M¹/L@M²) & $-0.85 /-0.77$ & -0.89 & -0.85 & $-0.45 /-0.05$ & $-0.42 /-0.04$ \\
\hline $\mathrm{CT}^{[\mathrm{d}]} \mathrm{LM}^{2} \rightarrow \mathrm{LM}^{1}$ & 0.00 & & & 0.41 & 0.44 \\
\hline
\end{tabular}

[a] Distances in $\AA$, angles in degree, charges in e.

[b] Dimer extracted from x-ray structure, not optimized. 
[c] Charges derived by NPA scheme of the metal atoms $\left(\mathrm{M}^{1}, \mathrm{M}^{2}\right)$, the ligands $(L)$ and the complexes $\left(M^{1}-L\right.$ and $\left.M^{2}-L\right)$ in the TPPM complexes and TPPM ${ }^{1 / T P P M}{ }^{2}$ dimers. The charges of the dimeric complexes sum up to the total charge of the dimer (neutral TPPCu/TPPCr and cationic LCu/LRu for $L=$ Por, TPP).

[d] Amount of charge transfer (CT) from $\mathrm{LM}^{2}$ to $\mathrm{LM}^{1}$ in e as determined via NPA.

In conclusion, we have undertaken the first experimental and theoretical steps toward achieving a self-doping mixed metalloporphyrin system. We learned that 1 ) for smooth electron transfer, it is necessary to use two transition metals which differ notably in their redox characteristics, that 2) the stacked arrangement is preferred either by "stackable" ligands or by fixing the porphyrin, phthalocyanine or corrole entities closer together, possibly in a polymeric stacked manner, ${ }^{5}$ and that 3) a sound theoretical treatment requires sophisticated quantum mechanical techniques beyond DFT. These challenges are worth pursuing in future work, because a wide variety of non-innocent ligands ${ }^{1-3}$ with different transition metals can be envisioned with potential applications as electronic materials and catalysts.

\section{Conflicts of interest}

There are no conflicts to declare.

\section{Acknowledgements}

We thank Udo Lehmann and Rainer Krömer for initial experiments.

\section{Notes and references}

1 Reviews and selected papers of porphyrins: a) K. M. Kadish, K. M. Smith and R. Guilard (eds.), The Porphyrin Handbook, Volumes 1-20, Academic Press, New York, 1999-2003; b) E. Aguilar-Ortiz, A. R. Jalilian and M. A. Avila-Rodriguez, MedChemCommun 2018, 9, 1577-1588; c) L. Smykalla, C. Mende, M. Fronk, P. F. Siles, M. Hietschold, G. Salvan, D. R.

T. Zahn, O. G. Schmidt, T. Rüffer and H. Lang, Beilstein J. Nanotechnol. 2017, 8, 17861800; d) S. Yamashita, T. Imamura and Y. Sasaki, Chem. Lett. 1995, 24, 417-418; e) M. Tsutsui, R. A. Velapoldi, K. Suzuki, F. Vohwinkel, M. Ichikawa and T. Koyano, J. Am. Chem. Soc. 1969, 91, 6262-6266; f) K. M. Smith, New J. Chem. 2016, 40, 5644-5649.

2 Reviews and selected papers of metallophthalocyanines: a) C. C. Leznoff and A. B. P. Lever (eds.), Phthalocyanines - Properties and Applications, VCH, New York, 1996; b) A. B. P. Lever and J. P. Wilshire, Can J. Chem. 1976, 54, 2514-2516; c) S. Yoshimoto, J. Porph. Phthalocyanines 2007, 11, 313-333; d) J. Zhou and Q. Sun, J. Am. Chem. Soc. 
2011, 133, 15113-15119; e) M. Hanack, Molecules 2015, 20, 20173-20185; f) A. C. Y. Tay, B. J. Frogley, D. C. Ware, J. Conradie, A. Ghosh and P. J. Brothers, Angew. Chem. Int. Ed. 2019, 58, 3057-3061; g) I. M. Denekamp, F. L. P. Veenstra, P. Jungbacker and G. Rothenberg, Appl. Organometal. Chem. 2019, 33: e4872.

3 Selected papers of metallocorroles: a) S. Ganguly and A. Ghosh, Acc. Chem. Res. 2019, 52, 2003-2014; b) W. Sinha, A. Mahammed, N. Fridman, Y. Diskin-Posner, L. J. W. Shimon and Z. Gross, Chem. Commun. 2019, 55, 11912-11915; c) H. Lim, K. E. Thomas, B. Hedman, K. O. Hodgson, A. Ghosh and E. I. Solomon, Inorg. Chem. 2019, 58, 6722-6730; d) S. Nardis, F. Mandoj, M. Stefanelli and R. Paolesse, Coord. Chem. Rev. 2019, 388, 360405; e) K. M. Kadish, L. Fremond, Z. Ou, J. Shao, C. Shi, F. C. Anson, F. Burdet, C. P. Gros, J.-M. Barbe and R. Guilard, J. Am. Chem. Soc. 2005, 127, 5625-5631.

4 a) J. M. Gottfried, Surf. Sci. Reports 2015, 70, 259-379; b) T. Basova, A. Hassan, M. Durmus, A. G. Gürek and V. Ahsen, Coord. Chem. Rev. 2016, 310, 131-153.

5 Selected key papers and reviews of metalloporphyrin-based catalysis: a) Y. Liu, G. Zhou, Z. Zhang, H. Lei, Z. Yao, J. Li, J. Lin and R. Cao, Chem. Sci. 2020, 11, 87-96; b) G. Li, P. A. Kates, A. K. Dilger, P. T. Cheng, W. R. Ewing and J. T. Groves, ACS Catal. 2019, 9, 95139517; c) K. C. Ranjeesh, L. George, V. C. Wakchaure, Goudappagouda, R. N. Devi and S. S. Babu, Chem. Comm. 2019, 55, 1627-1630; d) Y. Guo, W. Shi, H. Yang, Q. He, Z. Zeng, J. Y. Ye, X. He, R. Huang, C. Wang and W. Lin, J. Am. Chem. Soc. 2019, 141, 17875-17883; e) D. Yang, H. Yu, T. He, S. Zuo, X. Liu, H. Yang, B. Ni, H. Li, L. Gu, D. Wang and X. Wang, Nat. Comm. 2019, 10: 3844; f) Y. Yang, G. Li, X. Mao and Y. She, Org. Process Res. Dev. 2019, 23, 1078-1086; g) Y. Hu, K. Lang, J. Tao, M. K. Marshall, Q. Cheng, X. Cui, L. Wojtas and X. P. Zhang, Angew. Chem. Int. Ed. 2019, 58, 2670-2674; h) B. Mondal, P. Sen, A. Rana, D. Saha, P. Das and A. Dey, ACS Catal. 2019, 9, 3895-3899; i) W. Wang, C. Li, J. Jin, L. Yan and Y. Ding, Dalton Trans. 2018, 47, 13135-13141; j) W. Zhang, W. Lai and R. Cao, Chem. Rev. 2017, 117, 3717-3797; k) C. Römelt, J. Song, M. Tarrago, J. A. Rees, M. Van Gastel, T. Weyhermüller, S. Debeer, E. Bill, F. Neese and S. Ye, Inorg. Chem. 2017, 56, 47454750; B. Meunier and J. Bernadou, Top. Catal. 2002, 21, 47-54; m) J. P. Collman, X. Zhang, V. J. Lee, E. S. Uffelman and J. I. Brauman, Science 1993, 261, 1404-1411; n) J. T. Groves and P. Viski, J. Org. Chem. 1990, 55, 3628-3634.

6 T. J. Marks, Science 1985, 227, 881-889.

7 H. Shirakawa, E. J. Louis, A. G. MacDiarmid, C. K. Chiang and A. J. Heeger, J. Chem. Soc. Chem. Commun. 1977, 578-580.

8 U. Lehmann, Dissertation, Phillips-Universität Marburg/ Germany, 1994.

9 M. J. Hamor, T. A. Hamor and J. L. Hoard, J. Am. Chem. Soc. 1964, 86, 1938-1942.

10 N. Li, Z. Su, P. Coppens and J. Landrum, J. Am. Chem. Soc. 1990, 112, 7294-7298.

11 P. Madura and W. R. Scheidt, Inorg. Chem. 1976, 15, 3182-3184. 
12 a) E. B. Fleischer, C. K. Miller and L. E. Webb, J. Am. Chem. Soc. 1964, 86, 2342-2347; b) A. L. Maclean, G. J. Foran, F. J. Kennedy, P. Turner and T. W. Hambley, Aust. J. Chem. 1996, 49, 1273-1278; c) Zhen Li, Yali Hu and Tingbin Li, Mol. Cryst. Liq. Cryst. 2014, 605, 135-145.

13 C. Chen, X.-T. He, D.-L. Hong, J.-W. Wang, Y.-H. Luo and B.-W. Sun, New J. Chem. 2018, 42, 12570-12575.

14 A. Hazell, Acta Crystallogr. 1984, C40, 751-753.

15 The lithium atom is square-planar coordinated to the four nitrogen atoms, but the porphyrin macrocycle is slightly distorted in the solid and adopts a wav conformation: A. Gebauer, D. Y. Dawson and J. Arnold, J. Chem. Soc., Dalton Trans. 2000, 111-112.

16 W. R. Scheidt, J. U. Mondal, C. W. Eigenbrot, A. Adler, L. J. Radonovich and J. L. Hoard, Inorg. Chem. 1986, 25, 795-799.

17S. D. Hoffmann, T. F. Faessler and J. W. Buchler, Cambridge Structural Database, Private Communication, 2010.

18 A. Hazell, Acta Crystallogr. 1986, C42, 296-299.

19S. Prei, C. Forster, Sven Otto, M. Bauer, P. Muller, D. Hinderberger, H. H. Haeri, L. Carrella and K. Heinze, Nature Chemistry 2017, 9, 1249-1254.

20 L. Aparici Plaza and J. Chojnacki, Acta Crystallogr. 2012, C68, m24-m28.

21 M. P. Byrn, C. J. Curtis, Yu. Hsiou, S. I. Khan, P. A. Sawin, S. K. Tendick, A. Terzis and C. E. Strouse, J. Am. Chem. Soc. 1993, 115, 9480-9497.

22 S. J. Silvers and A. Tulinsky, J. Am. Chem. Soc. 1967, 89, 3331-3337.

23 G. Donnay and C. B. Storm, Mol. Cryst. 1967, 2, 287-291.

24 W. R. Scheidt and C. A. Reed, Inorg. Chem. 1978, 17, 710-714.

25 H.-S. He, Acta Crystallogr. 2007, E63, m976-m977.

26 J. A. Cissell, T. P. Vaid and G. P. A. Yap, J. Am. Chem. Soc. 2007, 129, 7841-7847.

27 M. L. Schneider, J. Chem. Soc., Dalton Trans. 1972, 1093-1096.

28 B. Cordero, V. Gómez, A. E. Platero-Prats, M. Revés, J. Echeverría, E. Cremades, F. Barragán and S. Alvarez, Dalton Trans. 2008, 21, 2832-2838.

29 C. Zhuo, C. Ou, C. Hu and J. Lang, J. Porphyrins Phthalocyanines 2017, 21, 211-221.

30 C.-H. Yang, S. J. Dzugan and V. L. Goedken, J. Chem. Soc., Chem Commun. 1986, 13131315.

31 D. V. Konarev, S. S. Khasanov, G. Saito, R. N. Lyubovskaya, Y. Yoshida and A. Otsuka, Chem. Eur. J. 2003, 9, 3837-3848.

32 a) J. P. Perdew, Phys. Rev. B 1986, 33, 8822-8824; b) A. Becke, Phys. Rev. A 1988, 38, 3098-3100; c) F. Weigend and R. Ahlrichs, Phys. Chem. Chem. Phys. 2005, 7, 3297-3305; d) S. Grimme, J. Antony, S. Ehrlich and H. Krieg, J. Chem. Phys. 2010, 132, 154104; e) S. Grimme, S. Ehrlich and L. Goerigk, J. Comput. Chem. 2011, 32, 1456-1465; f) A. E. Reed, R. B. Weinstock and F. Weinhold, J. Chem. Phys. 1985, 83, 735-746. 
33 C. R. Groom, I. J. Bruno, M. P. Lightfoot and S. C. Ward, Acta Cryst. 2016. B72, 171-179. 34 P. Doppelt, J. Fischer and R. Weiss, Inorg. Chem. 1984, 23, 2958-2962.

35 T. Mashiko, C. A. Reed, K. J. Haller and W. R. Scheidt, Inorg. Chem. 1984, 23, 3192-3196. 